Pacific Journal of Mathematics

ON GROSS DIFFERENTIATION ON BANACH SPACES 


\title{
ON GROSS DIFFERENTIATION ON BANACH SPACES
}

\author{
HUI-HSIUNG Kuo
}

Let $p_{t}(x, \cdot)$ denote the Wiener measure in an abstract Wiener space $(H, B)$ with variance parameter $t>0$ and mean $x$ in $B$. It is shown that if $f \in L^{2} p_{t}(x, \cdot), t>0$ and $x$ are fixed, then the function $p_{t} f$ defined by $p_{t} f(x+h)=\int_{B} f(y) p_{t}(x+h$, $d y$ ) for $h$ in $H$ is infinitely Gross differentiable at $x$. The first two derivatives are given by $\left(D p_{t} f(x), h\right)=t^{-1} \int_{B} f(y)(h$, $y-x) p_{t}(x, d y)$ and $\left(D^{2} p_{t} f(x) k, h\right)=t^{-1} \int_{B} f(y)\left\{t^{-1}(h, y-x)(k\right.$, $y-x)-(h, k)\} p_{t}(x, d y)$, where $h$ and $k$ are in $H$. Moreover, $D^{2} p_{t} f(x)$ is a Hilbert-Schmidt operator and $\left\|D^{2} p_{t} f(x)\right\|_{2} \leqq$ $\sqrt{2} t^{-1}\left\{\int_{B}|f(y)|^{2} p_{t}(x, d y)\right\}^{1 / 2}$. An application to UhlenbeckOrnstein process is also given.

1. Introduction. It is well-known that in a general Banach space $B$ the Frechet differentiable functions on $B$ do not form a very large class of functions. The works [1; 7], among others, show that for many separable Banach spaces the bounded continuously Frechet differentiable functions are not dense in the space of bounded uniformly continuous functions. However, by regarding a real separable Banach space $B$ as an abstract Wiener space [3], Goodman [2] is able to show that the set of bounded continuously quasi-differentiable functions on $B$ is dense in the space of bounded uniformly continuous functions on $B$. Regarding $B$ as an abstract Wiener space has a more important advantage, namely, we can talk about whether the second derivative is a Hilbert-Schmidt or trace class operator.

Let $(H, B)$ be an abstract Wiener space. In [4], a real valued function $u$ defined in an open subset $V$ of $B$ is said to be $H$-differentiable at $x \in V$ if there is an element $y$ in $H$ such that $\mid u(x+h)-$ $u(x)-(y, h) \mid=o(|h|)$ for $h$ in $H$, where $|\cdot|$ and (,) are the norm and the inner product of $H$, respectively. $y$ is easily seen to be unique and is denoted by $D u(x)$. Higher derivatives are defined similarly. Let $p_{t}(x, \cdot)$ denote the Wiener measure in $(H, B)$ with variance parameter $t>0$ and mean $x \in B . \quad p_{t}(0, \cdot)$ will be written as $p_{t}(\cdot)$. If $f$ is a bounded measurable complex valued function on $B$, we define $p_{t} f(x)=\int_{B} f(x+y) p_{t}(d y)$.

THEOREM (Gross [4]). Let $f$ be a bounded measurable function on $B$. Then $p_{t} f$ is infinitely $H$-differentiable on $B$ with the first 
and second derivatives given by

$$
\begin{aligned}
\left(D p_{t} f(x), h\right) & =t^{-1} \int_{B} f(x+y)(h, y) p_{t}(d y), \\
\left(D^{2} p_{t} f(x) k, h\right) & =t^{-1} \int_{B} f(x+y)\left\{t^{-1}(h, y)(k, y)-(h, k)\right\} p_{t}(d y),
\end{aligned}
$$

where $h$ and $k$ are in $H$. If $T$ is a test operator then

$$
\operatorname{trace}\left[T D^{2} p_{t} f(x)\right]=t^{-1} \int_{B} f(x+y)\left\{t^{-1}\langle T y, y\rangle-\operatorname{trace} T\right\} p_{t}(d y),
$$

where $\langle$,$\rangle is the natural pairing between B^{*}$ and $B$. Moreover, $D^{2} p_{t} f(x)$ is a Hilbert-Schmidt operator and

$$
\left\|D^{2} p_{t} f(x)\right\|_{2} \leqq \sqrt{2} t^{-1}\left\{\int_{B}|f(x+y)|^{2} p_{t}(d y)\right\}^{1 / 2} .
$$

The assumption that $f$ is bounded makes the above theorem uneasy to apply. For instance, it is desirable to differentiate the function $r_{t} f$ defined by $r_{t} f(x)=\int_{B} f(y) r_{t}(x, d y)$, where $f \in L^{2}\left(p_{1}\right)$ and $r_{t}(x, \cdot)=p_{1-e^{-2 t}}\left(e^{-t} x, \cdot\right)$ are the transition probabilities of UhlenbeckOrnstein process. Since $f$ may not be bounded, the above theorem is not applicable. To overcome this difficulty Piech considers an orthonormal basis of $L^{2}\left(p_{1}\right)$ consisting of Hermite cylinder functions and proves the following theorem.

THEOREM (Piech [6]). Let $g \in L^{2}\left(p_{1}\right)$. Then for $p_{1}$-a.e. $x$ and for $t>0, r_{t} g$ is twice $H$-differentiable at $x$ with derivatives given by

$$
\begin{aligned}
\left(D r_{t} g(x), h\right)= & -\left[e^{t}\left(1-e^{-2 t}\right)\right]^{-1} \int_{B} g(y)\left(e^{-t} x-y, h\right) r_{t}(x, d y), \\
\left(D^{2} r_{t} g(x) h, k\right)= & \left(e^{2 t}-1\right)^{-1} \int_{B} g(y)\left\{\left(1-e^{-2 t}\right)^{-1}\left(e^{-t} x-y, h\right)\left(e^{-t} x-y, k\right)\right. \\
& -(h, k)\} r_{t}(x, d y) .
\end{aligned}
$$

Moreover, $\left|D r_{t} g\right|$ and $\left\|D^{2} r_{t} g\right\|_{2}$ are in $L^{2}\left(p_{1}\right)$.

In this paper, we will reexamine how the $H$-derivatives are defined by Gross in [4] and prove his formulas in a rather general situation which we believe will be quite useful in $L^{2}\left(p_{1}\right)$-theory associated with Uhlenbeck-Ornstein process as well as other processes in $B$. We will see that our theorem yields Piech's in a beautiful way. The novelties in our approach are Lemma 1, Theorem 2 and the way we rewrite the expressions in Gross' theorem. For instance, the first derivative will be rewritten as $t^{-1} \int_{B} f(y)(h, y-x) p_{t}(x, d y)$, which exist when $f \in L^{2}\left(p_{t}(x, \cdot)\right.$ since $(h, \cdot-x) \in L^{2}\left(p_{t}(x, \cdot)\right)$. 
Definition 1. Let $V$ be a subset of $B$ and $x$ in $V$. $V$ is called $H$-coset open at $x$ if there exists $\delta=\delta(x)>0$ such that $x+h \in V$ for all $|h|<\delta$ in $H . \quad V$ is called $H$-coset open if $V$ is $H$-coset open at every point of $V$.

Obviously, if $G$ is an open subset of $H$ then $z+G$ is $H$-coset open for any $z$ in $B$. An open subset of $B$ is also $H$-coset open because $B$-norm is weaker then $H$-norm. But there are other subsets which are $H$-coset open. For instance, let $\left(H, B_{0}\right)$ be another abstract Wiener space with $B_{0} \subset B$ then any open subset of $B_{0}$ is also $H$-coset open. Perhaps this is the reason why our theorem is easier to apply because whenever a function is defined on an $H$-coset open set then we can talk about Gross differentiation of $f$ defined below.

DEFINITION 2. Let $f$ be a function defined on an $H$-coset open subset $V$ of $B$. Let $x \in V$. If the function $v(h)=f(x+h)$ defined on some $H$-neighborhood of 0 is $k$ th $(k \geqq 1)$ Frechet differentiable at 0 , then we say that $f$ is kth Gross differentiable at $x$. The $j$ th Gross derivative $D^{j} f(x)$ of $f$ at $x$ is defined to be the $j$ th Frechet derivative of $v$ at 0 . (Hence $D^{j} f(x)$ is a $j$-linear form on $H, 1 \leqq$ $j \leqq k)$.

From now on, $t>0$ and $x \in B$ will be fixed through $\S 4$. Suppose $f \in L^{2}\left(p_{t}(x, \cdot)\right)$, it will follow from Lemma 1 that $f \in L^{1}\left(p_{t}(x+h, \cdot)\right)$ for all $h$ in $H$. Therefore, $p_{t} f(x+h)=\int_{B} f(y) p_{t}(x+h, d y)$ is a function defined on the $H$-coset open set $x+H$.

THEOREM 1. Let $f \in L^{2}\left(p_{t}(x, \cdot)\right)$. Then the function $p_{t} f$ defined on the $H$-coset open set $x+H$ is infinitely Gross differentiable at $x$. The first and second Gross derivatives are given by

$$
\begin{gathered}
\left(D p_{t} f(x), h\right)=t^{-1} \int_{B} f(y)(h, y-x) p_{t}(x, d y) \\
\left(D^{2} p_{t} f(x) k, h\right)=t^{-1} \int_{B} f(y)\left\{t^{-1}(h, y-x)(k, y-x)-(h, k)\right\} p_{t}(x, d y) .
\end{gathered}
$$

If $T$ is a test operator then

$$
\begin{aligned}
\operatorname{trace} T D^{2} p_{t} f(x)= & t^{-1} \int_{B} f(y)\left\{t^{-1}\langle T(y-x), y-x\rangle\right. \\
& -\operatorname{trace} T\} p_{t}(x, d y) .
\end{aligned}
$$

Moreover, $D^{2} p_{t} f(x)$ is a Hilbert-Schmidt operator of $H$ and

$$
\left\|D^{2} p_{t} f(x)\right\|_{2} \leqq \sqrt{2} t^{-1}\left\{\int_{B}|f(y)|^{2} p_{t}(x, d y)\right\}^{1 / 2} .
$$


The proof of this theorem will be given in $\S 4$. In $\S 2$ and $\S 3$ we will state some integral formulas and prove some lemmas necessary for the proof. We remark that the idea of the proof comes essentially from [5, Proposition 1]. In $\S 5$ we will apply our theorem to Uhlenbeck-Ornstein process and derive Piech's theorem.

2. Some integral formulas. In the following $h$ and $k$ are in $H$ and $J_{t}(h, z)=\exp \left\{\left[-|h|^{2}+2(h, z)\right] / 2 t\right\}, h \in H$ and $z \in B$.

F 1).

$$
\int_{B}(h, z)^{2} p_{t}(d z)=t|h|^{2} \text {. }
$$

F 2).

$$
\int_{B}(h, z)^{4} p_{t}(d z)=3 t^{2}|h|^{4} .
$$

F 3).

$$
\int_{B}(h, z)^{8} p_{t}(d z)=105 t^{4}|h|^{8} .
$$

$$
\int_{B} J_{t}(h, z)^{2} p_{t}(d z)=e^{|h|^{2 / t}}
$$

F 5).

$$
\int_{B} J_{t}(h, z)^{4} p_{t}(d z)=e^{6|h|^{2 / t}}
$$

F 6).

$$
\text { Let } \theta_{t}(r)=e^{6 r^{2} / t}-4 e^{3 r^{2} / t}+6 e^{r^{2} / t}-3, r \geqq 0 \text {. }
$$

$$
\text { Then } \int_{B}\left[J_{t}(h, z)-1\right]^{4} p_{t}(d z)=\theta_{t}(|h|) .
$$

3. Some lemmas. Recall that $t>0$ and $x \in B$ are fixed. Note that for $h$ in $H$ the distribution function of $(h, y-x)$ with respect to $p_{t}(x, d y)$ is the same as that of $(h, z)$ with respect to $p_{t}(d z)$. Hence if $\phi$ is any complex valued Borel measurable function on $R$ then $\int_{B} \phi((h, y-x)) p_{t}(x, d y)=\int_{B} \phi((h, z)) p_{t}(d z)$. This remark will play an important role in the following computations.

Lemma 1. If $f \in L^{2}\left(p_{t}(x, \cdot)\right)$ then $f \in L^{1}\left(p_{t}(x+h, \cdot)\right)$ for all $h$ in $H$. In fact,

$$
\int_{B}|f(y)| p_{t}(x+h, d y) \leqq e^{|h|^{2} / 2 t}\left\{\int_{B}|f(y)|^{2} p_{t}(x, d y)\right\}^{1 / 2} .
$$

Proof. By the translation formula for the Wiener measures,

$$
p_{t}(x+h, d y)=J_{t}(h, y-x) p_{t}(x, d y) .
$$




$$
\begin{aligned}
& \int_{B}|f(y)| p_{t}(x+h, d y) \\
& \leqq\left\{\int_{B} J_{t}(h, y-x)^{2} p_{t}(x, d y)\right\}^{1 / 2}\left\{\int_{B}|f(y)|^{2} p_{t}(x, d y)\right\}^{1 / 2} \\
&=\left\{\int_{B} J_{t}(h, z)^{2} p_{t}(d z)\right\}^{1 / 2}\left\{\int_{B}|f(y)|^{2} p_{t}(x, d y)\right\}^{1 / 2} \\
&=e^{|h|^{2 / 2 t}}\left\{\int_{B}|f(y)|^{2} p_{t}(x, d y)\right\}^{1 / 2} .
\end{aligned}
$$

Here we have used the integral formula F4).

Lemma 2. If $f \in L^{2}\left(p_{t}(x, \cdot)\right)$ then for all $h$ and $k$ in $H, f(\cdot)(h$, $\cdot-x)$ and $f(\cdot)\left\{t^{-1}(h, \cdot-x)(k, \cdot-x)-(h, k)\right\}$ are in $L^{1}\left(p_{t}(x, \cdot)\right)$. In fact,

$$
\begin{aligned}
& \int_{B}|f(y)(h, y-x)| p_{t}(x, d y) \leqq \sqrt{t}|h|\left\{\int_{B}|f(y)|^{2} p_{t}(x, d y)\right\}^{1 / 2} . \\
& \int_{B}\left|f(y)\left\{t^{-1}(h, y-x)(k, y-x)-(h, k)\right\}\right| p_{t}(x, d y) \\
& \leqq(1+\sqrt{3})|h||k|\left\{\int_{B}|f(y)|^{2} p_{t}(x, d y)^{1 / 2}\right.
\end{aligned}
$$

Proof. Use F 1), F 2) and the Schwarz inequality.

Lemma 3. If $f \in L^{2}\left(p_{t}(x, \cdot)\right)$ then $f(\cdot)(h, \cdot-x) \in L^{1}\left(p_{t}(x+k, \cdot)\right)$ for all $h$ and $k$ in $H$. In fact,

$$
\begin{aligned}
& \int_{B}|f(y)(h, y-x)| p_{t}(x+k, d y) \\
& \quad \leqq \sqrt[4]{3} \sqrt{t}|h| e^{3|k|^{2 / 2 t}}\left\{\int_{B}|f(y)|^{2} p_{t}(x, d y)\right\}^{1 / 2}
\end{aligned}
$$

Proof.

$$
\begin{aligned}
& \int_{B}|f(y)(h, y-x)| p_{t}(x+k, d y) \\
&=\int_{B}|f(y)||(h, y-x)| J_{t}(k, y-x) p_{t}(x, d y) \\
& \leqq\left\{\int_{B}(h, y-x)^{4} p_{t}(x, d y)\right\}^{1 / 4}\left\{\int_{B} J_{t}(k, y-x)^{4} p_{t}(x, d y)\right\}^{1 / 4} \\
& \times\left\{\int_{B}|f(y)|^{2} p_{t}(x, d y)\right\}^{1 / 2} \\
&=\left\{\int_{B}(h, z)^{4} p_{t}(d z)\right\}^{1 / 4}\left\{\int_{B} J_{t}(k, z)^{4} p_{t}(d z)\right\}^{1 / 4}\left\{\int_{B}|f(y)|^{2} p_{t}(x, d y)\right\}^{1 / 2} \\
&= \sqrt[4]{3} \sqrt{t}|h| e^{3|k|^{2 / 2 t}}\left\{\int_{B}|f(y)|^{2} p_{t}(x, d y)\right\}^{1 / 2} .
\end{aligned}
$$


Here we have used integral formulas F2) and F5).

4. Proof of Theorem 1. By Lemma 1 the function $p_{t} f(x+h)$ is defined on the $H$-coset open subset $x+H$ of $B$. We divide the proof into several steps.

Step 1. To show that $p_{t} f$ is Gross differentiable and $\left(D p_{t} f(x)\right.$, $h)=t^{-1} \int_{B} f(y)(h, y-x) p_{t}(x, d y)$. (This integral exists by Lemma 2). Define $\phi(h)=p_{t} f(x+h)-p_{t} f(x)-t^{-1} \int_{B} f(y)(h, y-x) p_{t}(x, d y)$. We need to show that $|\phi(h)|=o(|h|)$ for small $|h|$. Let

$$
\begin{aligned}
& \phi_{1}(h)=t^{-1} \int f(y)(h, y-x) \int_{0}^{1}\left[J_{t}(s h, y-x)-1\right] d s p_{t}(x, d y), \\
& \dot{\phi}_{2}(h)=t^{-1} \int_{B} f(y) \int_{0}^{1} s|h|^{2} J_{t}(s h, y-x) d s p_{t}(x, d y) .
\end{aligned}
$$

Then by [4, p. 153] we have $\phi(h)=\phi_{1}(h)-\phi_{2}(h)$. But now we have to make better estimates.

$$
\begin{aligned}
t\left|\phi_{1}(h)\right| & \leqq \\
\leqq & \left.\int_{B}|f(y)|^{2} p_{t}(x, d y)\right\}^{1 / 2}\left\{\int_{B}(h, z)^{4} p_{t}(d z)\right\}^{1 / 4} \\
& \times\left\{\int_{0}^{1} \int_{B}\left[J_{t}(s h, z)-1\right]^{4} p_{t}(d z) d s\right\}^{1 / 4} \\
\leqq & \left\{\int_{B}|f(y)|^{2} p_{t}(x, d y)\right\}^{1 / 2}\left\{3 t^{2}|h|^{4}\right\}^{1 / 4}\left\{\int_{0}^{1} \theta_{t}(s|h|) d s\right\}^{1 / 4} \\
= & \sqrt[4]{3} \sqrt{t}|h|\left\{\int_{B}|f(y)|^{2} p_{t}(x, d y)\right\}^{1 / 2}\left\{\int_{0}^{1} \theta_{t}(s|h|) d s\right\}^{1 / 4} .
\end{aligned}
$$

Here we have used the integral formulas F2) and F6). It is easy to see that $\int_{0}^{1} \theta_{t}(s|h|) d s \rightarrow 0$ as $|h| \rightarrow 0$. Hence $\left|\phi_{1}(h)\right|=o(|h|)$ for small $|h|$. On the other hand,

$$
\begin{aligned}
t\left|\phi_{2}(h)\right| \leqq|h|^{2} \int_{0}^{1} s\left|\int_{B} f(y) J_{t}(s h, y-x) p_{t}(x, d y)\right| d s \\
=|h|^{2} \int_{0}^{1} s\left|\int_{B} f(y) p_{t}(x+s h, d y)\right| d s \\
\quad \leqq|h|^{2} \int_{0}^{1} s e^{s^{2}|h|^{2} / 2 t}\left\{\int_{B}|f(y)|^{2} p_{t}(x, d y)\right\}^{1 / 2} d s \\
=t\left(e^{|h|^{2} / 2 t}-1\right)\left\{\int_{B}|f(y)|^{2} p_{t}(x, d y)\right\}^{1 / 2}
\end{aligned}
$$

Hence $\left|\phi_{2}(h)\right|=o(|h|)$. Therefore, we have $|\phi(h)|=o(|h|)$. 
Step 2. To show that $p_{t} f$ is twice Gross differentiable and $\left(D^{2} p_{t} f(x) k, h\right)=t^{-1} \int_{B} f(y)\left\{t^{-1}(h, y-x)(k, y-x)-(h, k)\right\} p_{t}(x, d y)$.

It is easy to see that

$$
\begin{aligned}
\left(D p_{t} f(x+k), h\right)= & t^{-1} \int_{B} f(y)(h, y-x) J_{t}(k, y-x) p_{t}(x, d y) \\
& -t^{-1} \int_{B} f(y)(h, k) J_{t}(k, y-x) p_{t}(x, d y)
\end{aligned}
$$

Hence we have

$$
\begin{aligned}
&\left(D p_{t}\right.f(x+k), h)-\left(D p_{t} f(x), h\right)=t^{-1} \int_{B} f(y)(h, y-x) \\
& \quad \times\left[J_{t}(k, y-x)-1\right] p_{t}(x, d y)-t^{-1} \\
& \quad \times \int_{B} f(y)(h, k) J_{t}(k, y-x) p_{t}(x, d y)
\end{aligned}
$$

Note that

$$
J_{t}(k, y-x)-1=t^{-1} \int_{0}^{1}\left[(k, y-x)-s|k|^{2}\right] J_{t}(s k, y-x) d s .
$$

Therefore,

$$
\begin{aligned}
& \left(D p_{t} f(x+k), h\right)-\left(D p_{t} f(x), h\right)=t^{-2} \int_{B} f(y)(h, y-x)(k, y-x) \\
& \quad \times \int_{0}^{1} J_{t}(s k, y-x) d s p_{t}(x, d y)-t^{-2} \int_{B} f(y)(h, y-x) \\
& \quad \times \int_{0}^{1} s|k|^{2} J_{t}(s k, y-x) d s p_{t}(x, d y)-t^{-1}(h, k) \\
& \quad \times \int_{B} f(y) J_{t}(k, y-x) p_{t}(x, d y) .
\end{aligned}
$$

Now, define

$$
\begin{aligned}
\psi(h, k)= & \left(D p_{t} f(x+k), h\right)-\left(D p_{t} f(x), h\right) \\
& -t^{-1} \int_{B} f(y)\left\{t^{-1}(h, y-x)(k, y-x)-(h, k)\right\} p_{t}(x, d y), \\
\psi_{1}(h, k)= & t^{-2} \int_{B} f(y)(h, y-x)(k, y-x) \int_{0}^{1}\left[J_{t}(s k, y-x)-1\right] d s p_{t}(x, d y), \\
\psi_{2}(h, k)= & t^{-2} \int_{B} f(y)(h, y-x) \int_{0}^{1} s|k|^{2} J(s k, y-x) d s p_{t}(x, d y) \\
\psi_{3}(h, k)= & t^{-1}(h, k) \int_{B} f(y)\left[J_{t}(k, y-x)-1\right] p_{t}(x, d y) .
\end{aligned}
$$

Then by the above computation, we have $\psi(h, k)=\psi_{1}(h, k)+\psi_{2}(h, k)+$ $\psi_{3}(h, k)$. We will show that $\psi_{j}(h, k)=0(|h|) o(|k|)$ and our assertion 
in this step follows immediately.

$$
\begin{aligned}
& t^{2}\left|\psi_{1}(h, k)\right| \leqq\left\{\int_{B}|f(y)|^{2} p_{t}(x, d y)\right\}^{1 / 2}\left\{\int_{B}(h, y-x)^{8} p_{t}(x, d y)\right\}^{1 / 8} \\
& \times\left\{\int_{B}(k, y-x)^{8} p_{t}(x, d y)\right\}^{1 / 8}\left\{\int_{0}^{1} \int_{B}\left[J_{t}(s k, y-x)-1\right]^{4} p_{t}(x, d y) d s\right\}^{1 / 4} \\
&= \sqrt[4]{105} t|h||k|\left\{\int_{B}|f(y)|^{2} p_{t}(x, d y)\right\}^{1 / 2}\left\{\int_{0}^{1} \theta_{t}(s|k|) d s\right\}^{1 / 4} \\
&= o(|h|) o(|k|) . \\
& t^{2}\left|\psi_{2}(h, k)\right|=|k|^{2}\left|\int_{0}^{1} s \int_{B} f(y)(h, y-x) J_{t}(s k, y-x) p_{t}(x, d y) d s\right| \\
&=|k|^{2}\left|\int_{0}^{1} s \int_{B} f(y)(h, y-x) p_{t}(x+s k, d y) d s\right| \\
& \leqq|k|^{2} \int_{0}^{1} s \int_{B}|f(y)(h, y-x)| p_{t}(x+s k, d y) d s \\
& \leqq \sqrt[4]{3} \sqrt{t}|h||k|^{2}\left\{\int_{B}|f(y)|^{2} p_{t}(x, d y)\right\}^{1 / 2}\left\{\int_{0}^{1} s e^{\left.3 s^{2}|k|^{2 / 2 t} d s\right\}}\right. \\
&= \frac{1}{\sqrt[4]{27}} t^{3 / 2}|h|\left[e^{3|k|^{2} / 2 t}-1\right]\left\{\int_{B}|f(y)|^{2} p_{t}(x, d y)\right\}^{1 / 2} \\
&= O(|h|) o(|k|) .
\end{aligned}
$$

In the third step of estimating $\psi_{2}(h, k)$ we have used Lemma 3. Finally,

$$
\begin{aligned}
t\left|\psi_{3}(h, k)\right| & \leqq|h||k|\left\{\int_{B}|f(y)|^{2} p_{t}(x, d y)\right\}^{1 / 2}\left\{\int_{B}\left[J_{t}(k, y-x)-1\right]^{4} p_{t}(x, d y)\right\}^{1 / 4} \\
& =|h||k|\left\{\int_{B}|f(y)|^{2} p_{t}(x, d y)\right\}^{1 / 2}\left\{\theta_{t}(|k|\}^{1 / 4}\right. \\
& =O(|h|) o(|k|) .
\end{aligned}
$$

Step 3. Let $T$ be a test operator whose restriction to $H$ is symmetric. Then $T$ can be expressed as follows:

$$
T x=\sum_{j=1}^{n} \lambda_{j}\left\langle e_{j}, x\right\rangle e_{j},
$$

where $\langle$,$\rangle is the natural pairing between B^{*}$ and $B$, and $e_{j} \in B^{*}$, $j=1,2, \cdots, n$, are orthonormal. Then

$$
\begin{aligned}
\operatorname{trace} T D^{2} p_{t} f(x) & =\sum_{j=1}^{n}\left(T D^{2} p_{t} f(x) e_{j}, e_{j}\right) \\
& =\sum_{j=1}^{n} \lambda_{j}\left(D^{2} p_{t} f(x) e_{j}, e_{j}\right) \\
& =\sum_{j=1}^{n} t^{-1} \int_{B} f(y)\left\{t^{-1} \lambda_{j}\left\langle e_{j}, y-x\right\rangle^{2}-\lambda_{j}\right\} p_{t}(x, d y) \\
& =t^{-1} \int_{B} f(y)\left\{t^{-1}\langle T(y-x), y-x\rangle-\operatorname{trace} T\right\} p_{t}(x, d y) .
\end{aligned}
$$


Hence, for any symmetric test operator $T$,

$$
\begin{aligned}
\operatorname{trace} & T D^{2} p_{t} f(x) \\
= & t^{-1} \int_{B} f(y)\left\{t^{-1}\langle T(y-x), y-x\rangle-\operatorname{trace} T\right\} p_{t}(x, d y) .
\end{aligned}
$$

Observe that $D^{2} p_{t} f(x)$ is symmetric and that both sides of the above equality depend only on the symmetric part of $T$. Hence the above equality holds for all test operators. Moreover, this equality implies that

$$
\begin{aligned}
\mid \text { trace } & T D^{2} p_{t} f(x) \mid \\
& \leqq t^{-1}\left\{\int_{B}|f(y)|^{2} p_{t}(x, d y)\right\}^{1 / 2}\left\{\int _ { B } \left[t^{-1}\langle T(y-x), y-x\rangle\right.\right. \\
& \left.-\operatorname{trace} T]^{2} p_{t}(x, d y)\right\}^{1 / 2} \\
= & t^{-1}\left\{\int_{B}|f(y)|^{2} p_{t}(x, d y)\right\}^{1 / 2}\left\{\int_{B}\left[t^{-1}\langle T z, z\rangle-\operatorname{trace} T\right]^{2} p_{t}(d z)\right\}^{1 / 2} \\
= & t^{-1}\left\{\int_{B}|f(y)|^{2} p_{t}(x, d y)\right\}^{1 / 2} \sqrt{2}\|T\|_{2} .
\end{aligned}
$$

Hence, by the same argument as in [4, pp. 155-156], $D^{2} p_{t} f(x)$ is a Hilbert-Schmidt operator and

$$
\left\|D^{2} p_{t} f(x)\right\|_{2} \leqq \sqrt{2} t^{-1}\left\{\int_{B}|f(y)|^{2} p_{t}(x, d y)\right\}^{1 / 2}
$$

Step 4. To see the existence of the higher Gross derivatives of $p_{t} f$, observe that we need only the integrability with respect to $p_{t}(d z)$ of all polynomials in $(h, z)$ and in $J_{t}(h, z)$ for $h$ in $H$. It is easy to see that the method used in the previous computation yields the following estimate

$$
\begin{aligned}
& \mid D^{n} p_{t} f(x+k)\left(h_{1}, \cdots, h_{n}\right)-D^{n} p_{t} f(x)\left(h_{1}, \cdots, h_{n}\right) \\
& \quad-D^{n+1} p_{t} f(x)\left(h_{1}, \cdots, h_{n}, k\right) \mid=O\left(\left|h_{1}\right| \cdot\left|h_{2}\right| \cdots\left|h_{n}\right|\right) o(|k|),
\end{aligned}
$$

where $D^{n} p_{t} f(x)$ is the $n$th Gross derivative of $p_{t} f$ at $x, n \geqq 3$. Of course, we have to note that $D^{n} p_{t} f(x+k)$ is also a continuous $n$ linear form on $H$ for all $k$ in $H$.

5. An application to Uhlenbeck-Ornstein process. Let $r_{t}(x$, $d y)=p_{1-e^{-2 t}}\left(e^{-t} x, d y\right), t>0$ and $x \in B . \quad\left\{r_{t}(x, \cdot) ; t>0, x \in B\right\}$ generate a Markov process with continuous sample paths known as UhlenbeckOrnstein process. It is easy to check that $p_{1}$ is an invariant measure for $r_{t}(x, \cdot)$ for all $t>0$. That is, for all Borel subsets $A$ of $B$, 


$$
\int_{B} r_{t}(x, A) p_{1}(d x)=p_{1}(A), t>0 .
$$

THEOREM 2. Let $g \in L^{2}\left(p_{1}\right)$. Then for each $t>0$ there exists a measurable subset $B_{t}$ of $B$ such that $p_{1}\left(B_{t}\right)=1$ and $g \in L^{2}\left(r_{t}(x, \cdot)\right.$ for all $x$ in $B_{t}$.

REMARK. In a less precise way the above theorem says that if $g \in L^{2}\left(p_{1}\right)$ then $g \in L^{2}\left(r_{t}(x, \cdot)\right)$ for a.e. $x\left[p_{1}\right]$.

Proof. By Fubini's theorem and the invariance of $p_{1}$

$$
\begin{gathered}
\int_{B}\left[\int_{B}|g(y)|^{2} r_{t}(x, d y)\right] p_{1}(d x) \\
\quad=\int_{B}|g(y)|^{2} \int_{B} r_{t}(x, d y) p_{1}(d x) \\
\quad=\int_{B}|g(y)|^{2} p_{1}(d y)<\infty .
\end{gathered}
$$

Hence $g \in L^{2}\left(r_{t}(x, \cdot)\right)$ a.e. $x\left[p_{1}\right]$.

Let $g \in L^{2}\left(p_{1}\right)$. Then by the above theorem, we have $g \in L^{2}\left(r_{t}(x, \cdot)\right)$ for all $x \in B_{t}$. But

$$
\int_{B}|g(y)|^{2} r_{t}(x, d y)=\int_{B}|g(y)|^{2} p_{1-e^{-2 t}}\left(e^{-t} x, d y\right) .
$$

Hence $g \in L^{2}\left(p_{1-e^{-2 t}}\left(e^{-t} x, \cdot\right)\right)$ for all $x \in B_{t}$. Observe that $p_{1-e^{-2 t}} g\left(e^{-t} x\right)=$ $r_{t} g(x)$. Hence the formulas for first and second derivatives in Piech's theorem follow follow from Theorem 1. Moreover, by Theorem 1, for $x \in B_{t}$

$$
\begin{aligned}
\left\|D^{2} r_{t} g(x)\right\|_{2} & =e^{-2 t}\left\|D^{2} p_{1-e^{-2 t}} g\left(e^{-t} x\right)\right\|_{2} \\
& \leqq e^{-2 t} \sqrt{2}\left(1-e^{-2 t}\right)^{-1}\left\{\int_{B}|g(y)|^{2} p_{1-e^{-2 t}}\left(e^{-t} x, d y\right)\right\}^{1 / 2} \\
& =\sqrt{2}\left(e^{2 t}-1\right)^{-1}\left\{\int_{B}|g(y)|^{2} r_{t}(x, d y)\right\}^{1 / 2}
\end{aligned}
$$

Hence

$$
\begin{aligned}
\int_{B}\left\|D^{2} r_{t} g(x)\right\|_{2}^{2} p_{1}(d x) & =\int_{B_{t}}\left\|D^{2} r_{t} g(x)\right\|_{2}^{2} p_{1}(d x) \\
& \leqq 2\left(e^{2 t}-1\right)^{-2} \int_{B_{t}} \int_{B}|g(y)|^{2} r_{t}(x, d y) p_{1}(d x) \\
& =2\left(e^{2 t}-1\right)^{-2} \int_{B} \int_{B}|g(y)|^{2} r_{t}(x, d y) p_{1}(d x) \\
& =2\left(e^{2 t}-1\right)^{-2} \int_{B}|g(y)|^{2} p_{1}(d y) .
\end{aligned}
$$


On the other hand, it is easy to see that for $f$ in Theorem 1 we have

$$
\left|D p_{t} f(x)\right| \leqq t^{-1 / 2}\left\{\int_{B}|f(y)|^{2} p_{t}(x, d y)\right\}^{1 / 2}
$$

Hence by the same argument above

$$
\left|D r_{t} g(x)\right| \leqq\left(e^{2 t}-1\right)^{-1 / 2}\left\{\int_{B}|g(y)|^{2} r_{t}(x, d y)\right\}^{1 / 2}, x \in B_{t},
$$

and

$$
\int_{B}\left|D r_{t} g(x)\right|^{2} p_{1}(d x) \leqq\left(e^{2 t}-1\right)^{-1} \int_{B}|g(y)|^{2} p_{1}(d y) .
$$

The author is grateful to Professor M. Ann Piech for correcting a mistake, i.e., the constant factor $\sqrt{2}$ in the estimate $\left\|D^{2} p_{t} f(x)\right\|_{2}$ is missing in the original manuscripts.

\section{REFERENCES}

1. R. Bonic and J. Frampton, Differentiable functions on certain Banach spaces, Bull. Amer. Math. Soc., 71 (1965), 393-395.

2. V. Goodman, Quasi-differentiable functions on Banach spaces, Proc. Amer. Math. Soc., 30 (1971), 367-370.

3. L. Gross, Abstract Wiener spaces, Proc. Fifth Berkeley Sympos. Math. Statist. and Probability, vol. II, part 1 (1965), 31-42.

4. - Potential theory on Hilbert space, J. Functional Analysis, 1 (1967), 123-181.

5. H.-H. Kuo, Iniegration by parts for abstract Wiener measures, Duke Math. J., 41 (1974), 373-379.

6. M. Ann Piech, The Ornstein-Uhlenbeck semigroup in an infinite dimensional $L^{2}$ setting, J. Functional Analysis, 18 (1975), 271-285.

7. J. H. M. Whitfield, Differentiable functions with bounded nonempty support on Banach spaces, Bull. Amer. Math. Soc., 72 (1966), 145-146.

Received March 7, 1975. Research supported in part by NSF Grant GP-38010.

UNIVERSITY OF VIRGINIA 



\section{PACIFIC JOURNAL OF MATHEMATICS}

\section{EDITORS}

RICHARD ARENS (Managing Editor) University of California

Los Angeles, California 90024

\section{R. A. Beaumont}

University of Washington Seattle, Washington 98105
J. DugundJI Department of Mathematics University of Southern Californıa Los Angeles, California 90007

D. Gilbarg aNd J. Milgram Stanford University Stanford, California 94305

\section{ASSOCIATE EDITORS}

E. F. BeCKENBACH

B. H. NeUmanN

F. Wolf

K. YoSHIDA

\section{SUPPORTING INSTITUTIONS}

UNIVERSITY OF BRITISH COLUMBIA CALIFORNIA INSTITUTE OF TECHNOLOGY UNIVERSITY OF CALIFORNIA MONTANA STATE UNIVERSITY UNIVERSITY OF NEVADA NEW MEXICO STATE UNIVERSITY OREGON STATE UNIVERSITY UNIVERSITY OF OREGON OSAKA UNIVERSITY
UNIVERSITY OF SOUTHERN CALIFORNIA STANFORD UNIVERSITY UNIVERSITY OF TOKYO UNIVERSITY OF UTAH WASHINGTON STATE UNIVERSITY UNIVERSITY OF WASHINGTON AMERICAN MATHEMATICAL SOCIETY NAVAL WEAPONS CENTER 


\section{Pacific Journal of Mathematics \\ Vol. 59, No. 1 \\ May, 1975}

Shashi Prabha Arya and M. K. Singal, More sum theorems for topological

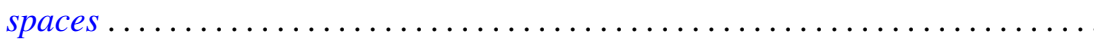

Goro Azumaya, F. Mbuntum and Kalathoor Varadarajan, On M-projective and

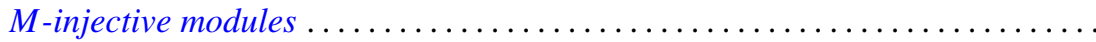

Kong Ming Chong, Spectral inequalities involving the infima and suprema of

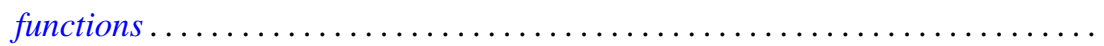

Alan Hetherington Durfee, The characteristic polynomial of the monodromy .......

Emilio Gagliardo and Clifford Alfons Kottman, Fixed points for orientation

preserving homeomorphisms of the plane which interchange two points ......

Raymond F. Gittings, Finite-to-one open maps of generalized metric spaces .......

Andrew M. W. Glass, W. Charles (Wilbur) Holland Jr. and Stephen H. McCleary,

$a^{*}$-closures of completely distributive lattice-ordered groups .............

Matthew Gould, Endomorphism and automorphism structure of direct squares of

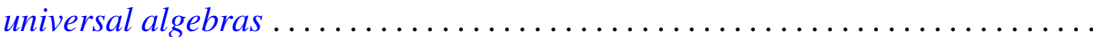

R. E. Harrell and Les Andrew Karlovitz, On tree structures in Banach spaces .....

Julien O. Hennefeld, Finding a maximal subalgebra on which the two Arens

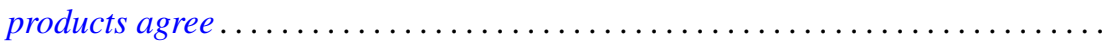

William Francis Keigher, Adjunctions and comonads in differential algebra .......

Robert Bernard Kelman, A Dirichlet-Jordan theorem for dual trigonometric

series

Allan Morton Krall, Stieltjes differential-boundary operators. III. Multivalued operators-linear relations...

Hui-Hsiung Kuo, On Gross differentiation on Banach spaces .

Tom Louton, A theorem on simultaneous observability ...

Kenneth Mandelberg, Amitsur cohomology for certain extensions of rings of algebraic integers.

Coy Lewis May, Automorphisms of compact Klein surfaces with boundary . . .

Peter A. McCoy, Generalized axisymmetric elliptic functions .

211

Muril Lynn Robertson, Concerning Siu's method for solving $y^{\prime}(t)=F(t$, $y(g(t)))$. .

Richard Lewis Roth, On restricting irreducible characters to normal

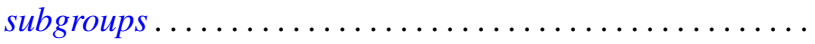

Albert Oscar Shar, $P$-primary decomposition of maps into an $H$-space .

Kenneth Barry Stolarsky, The sum of the distances to certain pointsets on the unit circle.

Bert Alan Taylor, Components of zero sets of analytic functions in $C^{2}$ in the unit ball or polydisc

Michel Valadier, Convex integrands on Souslin locally convex spaces ...

Januario Varela, Fields of automorphisms and derivations of $C$

Arnold Lewis Villone, A class of symmetric differential operators with deficiency

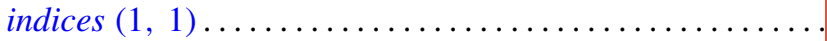

